Widefield Science ANd Technology for the SKA

SKADS CONFERENCE 2009

S.A. Torchinsky, A. van Ardenne, T. van den Brink-Havinga, A.J.J. van Es, A.J. Faulkner (eds.)

4-6 November 2009, Château de Limelette, Belgium

\title{
Some results from the BEST demonstrator ${ }^{\star}$
}

\author{
S. Montebugnoli ${ }^{1}$, F. Perini ${ }^{1}$, G. Bianchi ${ }^{1}$, P. Bolli ${ }^{2}$, G. Pupillo ${ }^{3}$, G. Naldi ${ }^{4}$, M. Schiaffino ${ }^{1}$, and J. Monari ${ }^{1}$ \\ 1 I.N.A.F. - I.R.A, Via Fiorentina, 3508/B 40059 Medicina, Italy \\ email: s.montebugnoli@ira.inaf.it,f.perini@ira.inaf.it, g.bianchi@ira.inaf.it, \\ mschiaffino@med.ira.inaf.it, j.monari@ira.inaf.it \\ 2 INAF-Osservatorio Astronomico di Cagliari, Loc. Poggio dei Pini, Strada 54, 09012 Capoterra (CA), Italy \\ email: pbolli@ca.astro.it \\ 3 INAF-Osservatorio Astronomico di Torino, Strada Osservatorio 20, 10025, Pino Torinese (TO), Italy \\ email: g.pupillo@med.ira.inaf.it \\ ${ }^{4}$ Università degli Studi di Bologna, Dipartimento di Astronomia, Via Ranzani 1, 40127 Bologna, Italy \\ email: gnaldi@med.ira.inaf.it
}

\begin{abstract}
In this contribution a collection of significant results from the BEST (Basic Element for SKA Training). demonstrator, based on cylindrical concentrators, is reported. Both technological deliverables and preliminary observation tests are available for further works and investigation on low frequency array.
\end{abstract}

\section{Introduction}

A $1400 \mathrm{~m}^{2}$ test bed, based on existing cylindrical concentrators of the Northern Cross array, has been prepared in the frame of the FP-6 SKADS program. Due to the velocity, programmability of the data acquisition system and the collecting area, the BEST prototype allowed to perform some beamforming activities and several preliminary astronomical tests. Since a lot of very strong RFIs were present into the new 400-416 MHz operational band, a very valuable check of the dynamic range in both the analogue and digital dominions were possible.

\section{Evaluation of the antenna noise temperature}

With BEST-1, the first in time SKADS technological demonstrator, based on the re-instrumentation of a single N/S cylindrical reflector of the Northern Cross radio telescope, we checked on the field the antenna temperature distribution calculation proposed by the International SKA Project Office, as a guideline to compare all the SKA antenna sensors (Bolli et al. 2008). The simulated antenna temperature, $T_{\text {ant }}$ (see Eq. 1), was obtained through an ad hoc Fortran code, which integrated the proposed brightness temperature distribution $T_{\mathrm{b}}$ (Cortes 2007), with the BEST-1 power pattern $P_{\mathrm{n}}$, obtained through GRASP simulations (see Fig. 1).

$$
T_{\text {ant }}\left(v ; \Theta_{0}, \Phi_{0}, \Delta_{0}\right)=\frac{\iint P_{n}(v ; \theta, \phi) T_{b}\left(v ; \theta^{\prime}, \phi^{\prime}\right) \sin \theta d \theta d \phi}{\iint P_{n}(v ; \theta, \phi) \sin \theta d \theta d \phi}
$$

The calculations were done at the BEST-1 central frequency $(v=408 \mathrm{MHz})$ and for various antenna pointing directions $\left(\Theta_{0}, \Phi_{0}, \Delta_{0}\right)$. In particular, since the Northern Cross is a transit telescope, we considered only the pointing in the co-elevation plane, from $\Theta_{0}=0^{\circ}$, which represent the pointing towards the

\footnotetext{
^ This work was supported by the European Commission Framework Program 6, Project SKADS, Square Kilometre Array Design Studies (SKADS), contract no 011938.
}

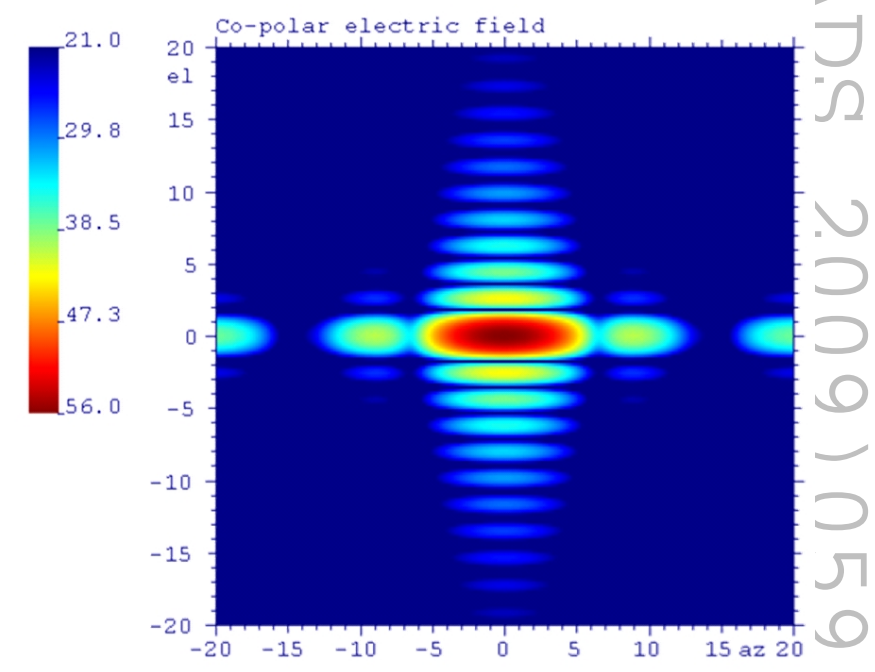

Fig. 1: Co-polar 2D BEST-1 main beam in elevation over azimuth plane

zenith direction, to $\Theta_{0}=80^{\circ}$ (see Fig. 2). The three curves are related to the contributions coming from the ground $\left(\theta^{\prime}>90^{\circ}\right.$, dashed curve), and from the sky $\left(\theta^{\prime} \leq 90^{\circ}\right.$, solid curve). Finally, the dash-dot line shows the total antenna temperature, which is the sum of the previous two contributions. In order to verify these numerical results, some experimental measurements were performed. The system noise temperature of BEST-1 (4 $\mathrm{RX}$ of a single N/S cylinder, summed together in order to obtain a single beam) was measured by detected transits of some strong calibrators (Cas-A and Virgo-A). Actually, the measurements allowed us to get the $A_{e f f} / T_{\text {sys }}$ figure. Hence, in order to determine $T_{\text {sys }}$, the effective area was evaluated as the product of the geometrical area and the antenna's efficiency. This last parameter was determined through the GRASP numerical 


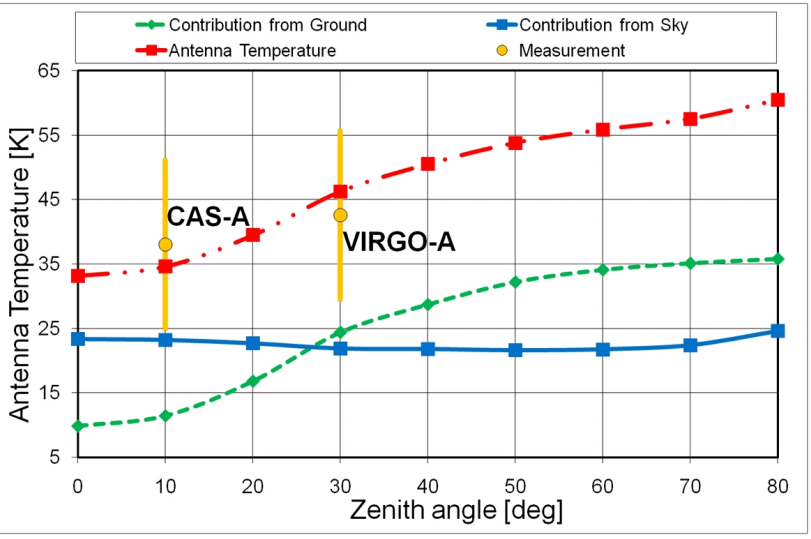

Fig. 2: BEST antenna temperature contributions against zenith angle
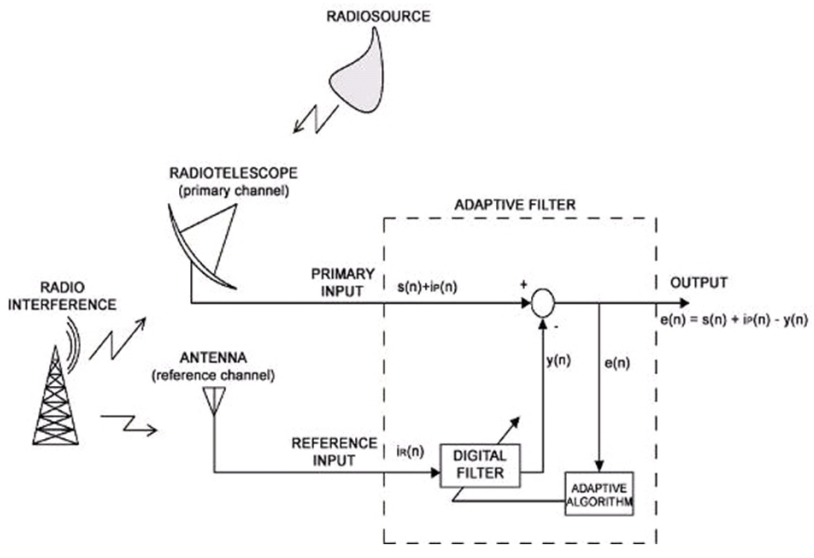

Fig. 3: MSC block diagram

analysis (the value turned out to be about $71 \%$ ), and not directly from measurement. We then estimated the antenna temperature from the system noise temperature using $T_{s y s}=T_{a n t}+T_{r e c}$, where $T_{\text {rec }}$ is the noise temperature of the receiver connected to the dipoles. For the previous formula, we measured a noise temperature equal to $47 \mathrm{~K}$, a value that also included the loss introduced by the transmission lines between the dipoles and the low-noise amplifiers. The measurements, reported with circles and vertical bars in Fig. 2, agreed very well with the numerical results, showing good accuracy in the proposed procedure. The measurement uncertainties depended on those of the calibrator fluxes, which were given within $10 \%$ to $15 \%$.

\section{RFI mitigation}

Different methods have been investigated using the BEST demonstrator. An algorithm tested is the MSC (Multiple Sidelobe Canceller). A block diagram is shown in Fig. 3. Some very preliminary results have been obtained as shown in Fig. 4 where a RFI signal generated by a meteorological balloon has been completely cancelled. This algorithm seems to work very well but the reference antenna has to be blind to the sky signal, otherwise it could be cancelled too.

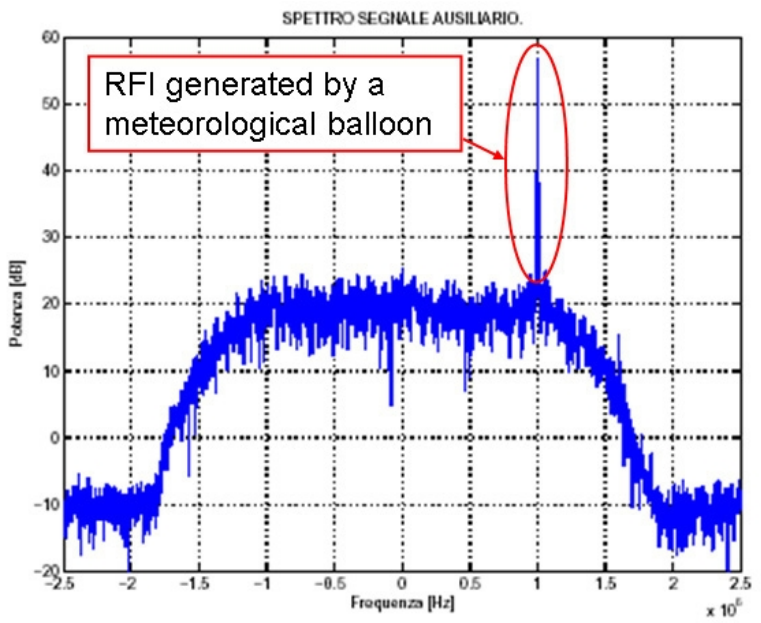

SPETIRO SEGIWE ASTRCNOAMOFILTRATO MSC.

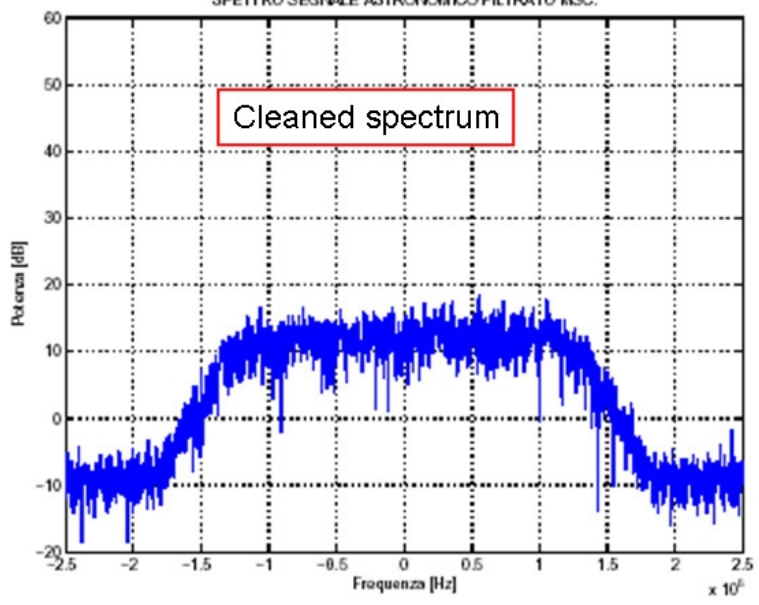

Fig. 4: MSC application to BEST-1 system: rejection of a meteorological stratospheric balloon

\section{A/D dynamic range evaluation}

A procedure to evaluate the number of bits, related to both resolution and dynamic range, was investigated (Bianchi et al. 2008). This procedure was then applied to the BEST system. The set up is composed of a spectrum analyser, a power meter and a logic analyser. The A/D converter is an Analog Devices AD6645 evaluation board. An ad hoc Labview program (running on a PC) to synchronize the instruments and acquire the data in the proper way has been designed. A picture of the measurement set up is shown in Fig. 5. This measurement phase lasted for a few weeks in order to acquire data from all the directions. Inside the $16 \mathrm{MHz}$ bandwidth of the BEST system, a lot of man-made signals were observed, especially radio relay stations. Sometimes a scientific stratospheric balloon is launched from a ground base station located close to the Medicina radio-telescope. The interference contribution from the balloon is variable and depends on its position due to the wind direction (Fig. 6). At the end of the measurement phase, 3 bit was the maximum value of the dynamic range variation observed (exclusively due to the interference contribution). Considering 3 bit for the sky-noise description, a 8 bit A/D con- 


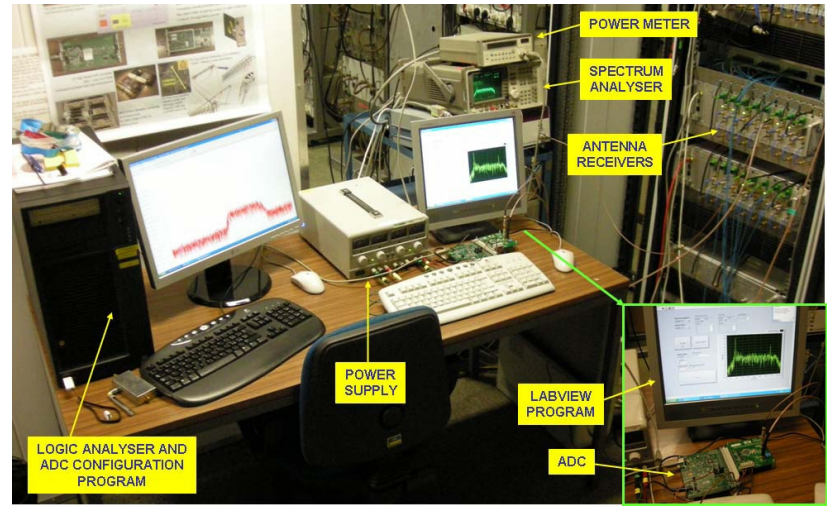

Fig. 5: Measurement bank

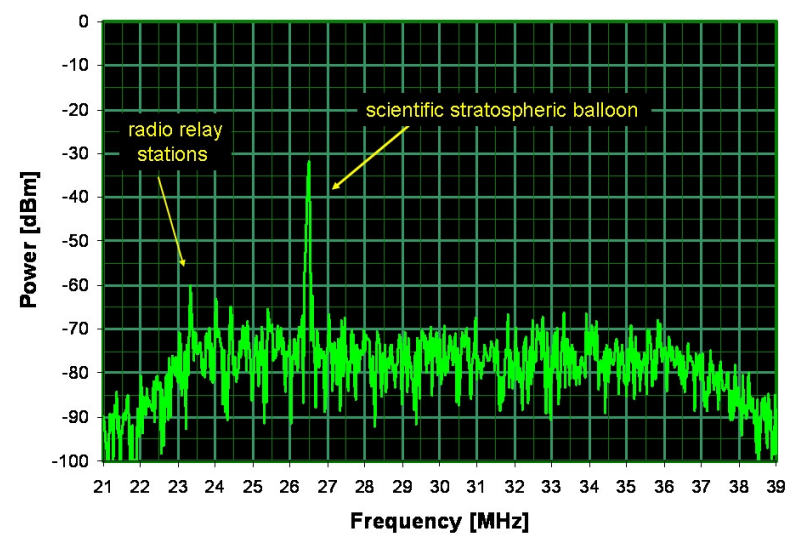

Fig. 6: BEST receiver power spectrum at the ADC input (IF)

verter, as the one installed on the ADC Casper board, has been considerate adequate.

\section{Phase stability of analogue optical front end}

In the BEST demonstrators, the selected receiver architecture are based on the antenna remotisation technique. In practice the RF signals are directly transported from the front ends, installed on the focal line of the antennas, down to a single main receiver room. This solution has been chosen following both reliability and costs analysis considerations that took into account different configurations of the whole system. The 3 stages RF front end and the optical TX operate without any temperature control. The optical fibre links are several hundred meters long, with some parts running on the antenna structure, some parts inside the cabins and some parts buried into the ground. In order to have a reliable stability test, we monitored for several days the receiver chain stability through astronomical observations. In practice, measuring the difference in time between the expected fringe transit time $\left(t_{\text {trans }}\right)$ and the measured one $\left(t_{\max }\right)$ in a simple interferometer $\left(\Delta t=t_{\max }-t_{\text {trans }}\right)$, it's possible to evaluate the instrumental phase $(\Delta \Phi)$. This is obtained determining the apparent source angular shift $\theta=\dot{\theta} \cdot \Delta t$, where $\dot{\theta}=(2 \pi / 86400) \cdot \cos \delta$ is the source angular velocity, and then

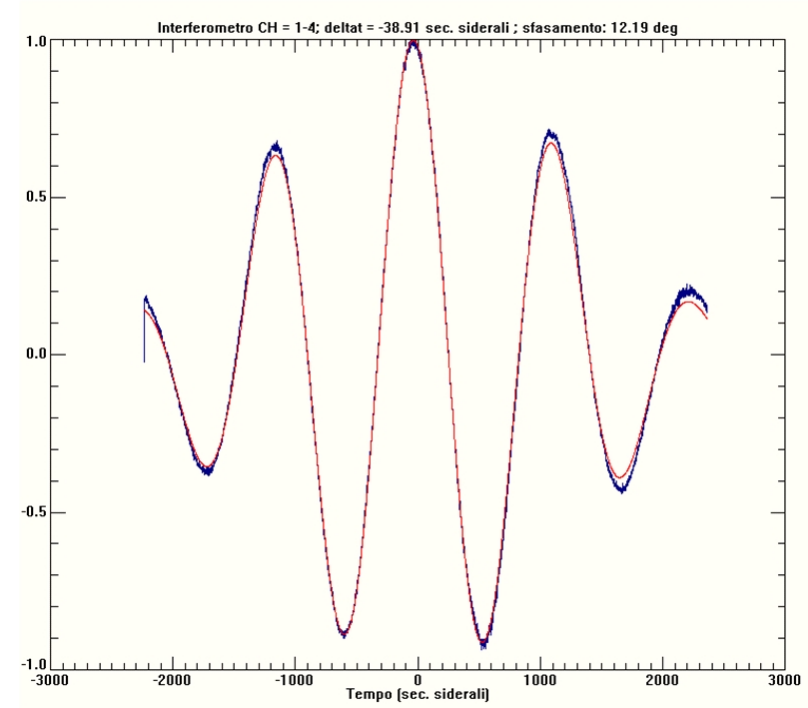

Fig. 7: Detected and fitted BEST fringes
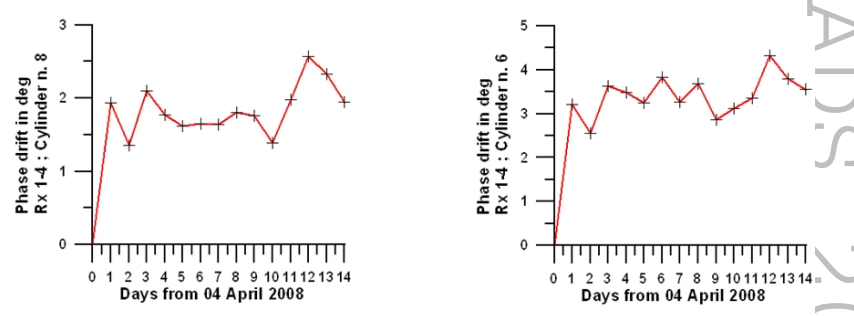

Fig. 8: BEST-2 RX phase $(\Delta \Phi)$ stability versus time

using Eq. 2:

$$
\Delta \Phi=-2 \pi \frac{\text { baseline }}{\lambda} \cdot \sin \theta
$$

The detected fringes (blue line in Fig. 7) are best-fitted (red line) with a simulated response in order to reduce the uncertainty on the transit time definition $\left(t_{\max }\right)$, in the case noise and/or RFI are present on the peaks of the fringes. Some results are reported in Fig. 8.

\section{Beamforming}

Some preliminary tests in the frame of the digital beamforming technique have been carried out in order to test the beam steering capability of the BEST array. In particular the architecture of a direct beamformer system has been implemented on the FPGA-based hardware of the Medicina digital back-end. Basically every analogue IF signal of the array is digitized by the A/D converter and down converted by a DDC; the resulting complex base band signal is then multiplied by the corresponding beamformer coefficient. Finally these signals are summed all together in order to form a single complex signal that represents the beam of the array. The beamformer output at the time $k, y(k)$, is calculated according to the following formula:

$$
y(k)=\boldsymbol{w}^{\boldsymbol{H}} \boldsymbol{v}(k)
$$




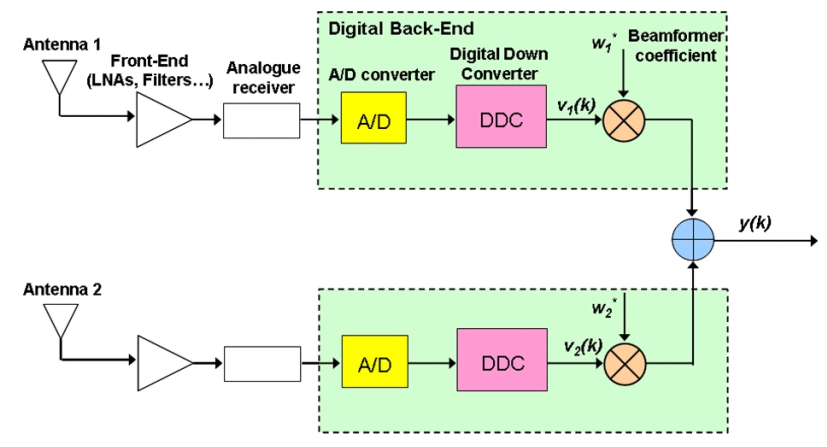

Fig. 9: Architecture of a two antennas beamformer

where $\boldsymbol{w}$ is the vector of the beamformer coefficients, $\boldsymbol{v}(k)$ is the vector of the complex baseband signals of the array at the time $k$ and $(\cdot)^{\boldsymbol{H}}$ is the Hermitian operator. For an array composed of two antennas the architecture of the system is illustrated in Fig. 9.

The effect of the phase correction introduced on the input signals by the beamformer coefficients has been tested with radio astronomical procedures. The architecture of the system used for these tests is the same of the one in Fig. 9, where the final adder block has been substituted by a multiplier block. The output signal is the result of the product correlation of two complex baseband signals (already multiplied by the beamformer coefficients). In practice, exploiting a radio source transit and analyzing the position in time of the measured interference fringe compared to the expected one, the phase corrections for every antenna are calculated and then applied to them. The results of the phase correction of two signals after Cassiopeia A (3C-461) transit is presented in Fig. 10. The two signals come from, respectively, the first and the fourth receiver of one cylinder of BEST-2 system. In the first experiment both signals are not phase shifted. With this design set up the maximum value of the observed interference fringe is in advance of about $38 \mathrm{~s}$. compared with the time at which the maximum value of the expected interference fringe happens with a calibrated array $(0 \mathrm{~s}$. in "Time from transit" axis). In order to correct this error (that is to calibrate the array) a phase of 12.19 degrees is applied to the second signal (fourth antenna) compared with the first one (first antenna): in other words the two signals are shifted in phase with an angle of 12.19 degrees. In this way the measured interference fringe matches perfectly with the expected one and its maximum value happens exactly at the theoretical transit time $(0 \mathrm{~s})$.

\section{Conclusions}

The BEST-1 and BEST-2 UHF prototypes represent a very good platform for preliminary tests to verify the main concepts on which the future SKA developments are based on. Very important calibration activities have been successfully carried on opening the possibility to move along the big technological challenge that will lead to the next step of SKA. The results from the BEST test bed seems to be very promising and useful as a feedback for other demonstrators.

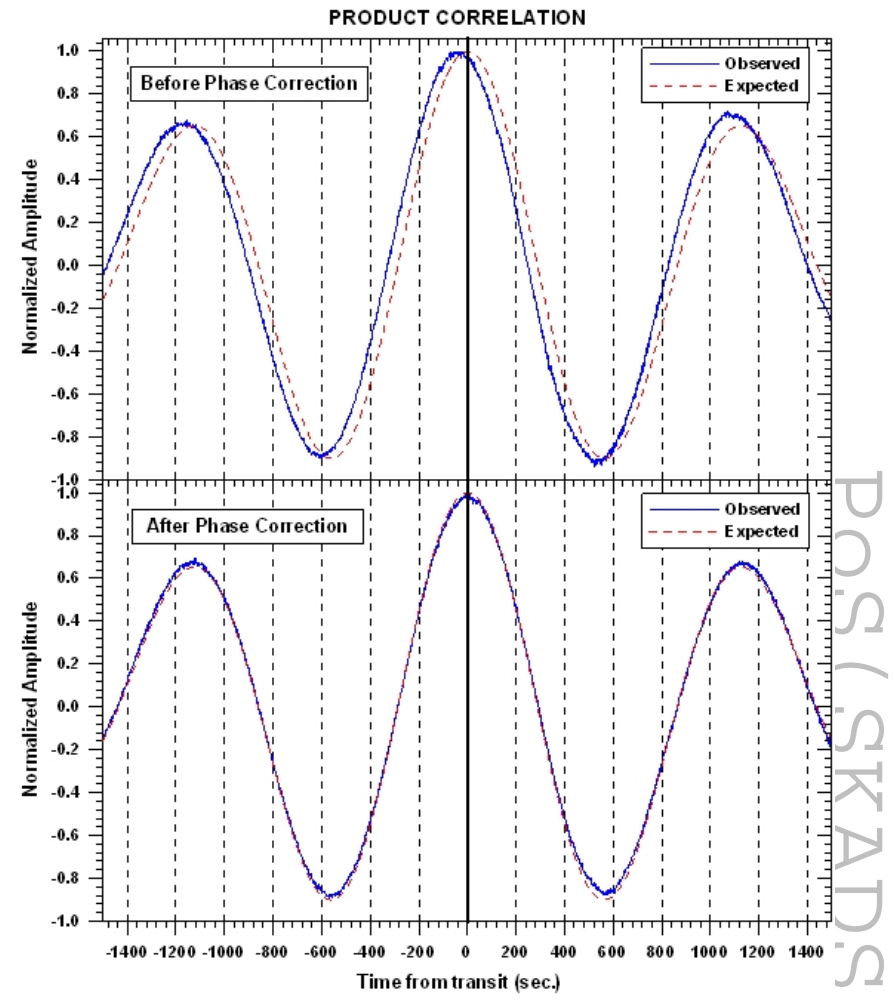

Fig. 10: Fringes with phase correction

\section{References}

Bianchi, G., F. Perini, C. Bortolotti, J. Monari, S. Montebugnoli, M. Roma, "ADC bit number and input power needed, in new radio-astronomical applications," Proceeding of $13^{\text {th }}$ Workshop on ADC Modelling and Testing, IMEKO TC4 - TC21 Joint Session, September $22^{\text {th }}$ - $24^{\text {th }} 2008$, Florence, Italy, p. 1064-1069

Bolli, P., F. Perini, S. Montebugnoli, G. Pelosi, S. Poppi, "Basic

Element for Square Kilometer Array Training (BEST):

Evaluation of the Antenna Noise Temperature," IEEE Antennas and Propagation Magazine, Vol.50, No. 2, April 2008

Cortes Medellin, G., "Antenna Noise Temperature Calculation," SKA Memo N.95 